\title{
Glutaminase 1 regulates the release of extracellular vesicles during neuroinflammation through key metabolic intermediate alpha-ketoglutarate
}

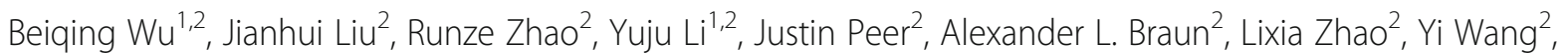
Zenghan Tong ${ }^{2}$, Yunlong Huang ${ }^{1,2^{*}}$ and Jialin C. Zheng ${ }^{1,2,3^{*}}$

\begin{abstract}
Background: Extracellular vesicles (EVs) are important in the intercellular communication of the central nervous system, and their release is increased during neuroinflammation. Our previous data demonstrated an increased release of EVs during HIV-1 infection and immune activation in glial cells. However, the molecular mechanism by which infection and inflammation increase EV release remains unknown. In the current study, we investigated the role of glutaminase 1 (GLS1)-mediated glutaminolysis and the production of a key metabolic intermediate aketoglutarate on EV release.

Methods: Human monocyte-derived macrophage primary cultures and a BV2 microglia cell line were used to represent the innate immune cells in the CNS. Transmission electron microscopy, nanoparticle tracking analysis, and Western blots were used to determine the EV regulation. GLS1 overexpression was performed using an adenovirus vector in vitro and transgenic mouse models in vivo. Data were evaluated statistically by ANOVA, followed by the Bonferroni post-test for paired observations.

Results: Our data revealed an increased release of EVs in GLS1-overexpressing HeLa cells. In HIV-1-infected macrophages and immune-activated microglia BV2 cells, treatment with bis-2-(5-phenylacetamido-1,2,4-thiadiazol-2-yl) ethyl sulfide (BPTES) or CB839, two specific GLS inhibitors, significantly decreased EV release, suggesting a critical role of GLS1 in EV release. Furthermore, addition of a-ketoglutarate or ceramide rescued EV release during BPTES treatment, implicating a-ketoglutarate and ceramide as critical downstream effectors for GLS inhibitors. These findings were further corroborated with the investigation of brain tissues in GLS1-transgenic mice. The EV levels were significantly higher in GLS1 transgenic mice than those in control mice, suggesting that GLS1 increases EV release in vivo.

Conclusions: These findings suggest that GLS1-mediated glutaminolysis and its downstream production of aketoglutarate are essential in regulating EV release during HIV-1 infection and immune activation. These new mechanistic regulations may help understand how glutamine metabolism shapes EV biogenesis and release during neuroinflammation.
\end{abstract}

Keywords: Glutamine metabolism, a-Ketoglutarate, Extracellular vesicles, HIV-1, Inflammation

\footnotetext{
* Correspondence: yhuan1@unmc.edu; jialinzheng@tongji.edu.cn

${ }^{1}$ Center for Translational Neurodegeneration and Regenerative Therapy,

Shanghai Tenth People's Hospital affiliated to Tongji University School of

Medicine, Shanghai, China

Full list of author information is available at the end of the article
} 


\section{Background}

Extracellular vesicles (EVs) are secretory vesicles budded from the plasma membrane of a variety of cells. EVs range from $50 \mathrm{~nm}$ to $1 \mathrm{~mm}$ and differ in their origins, either from direct fusion with plasma membrane or intracellular multivesicular bodies. EVs have been detected at an elevated level in the cerebral spinal fluid in patients with mild to severe Alzheimer's disease, Parkinson's disease, prion disease, and amyotrophic lateral sclerosis $[1,2]$. Protein markers of EVs are present in neuritic plaques in $\mathrm{AD}$ brains. Furthermore, increasing EV release likely contributes to the toxicity of amyloid beta and tau phosphorylation [3]. Therefore, EVs play a potential role in the pathogenesis of $\mathrm{AD}$. Mechanisms regulating EV release remain poorly understood. Recent studies indicate that the formation and secretion of EVs are largely dependent on the proper function of ceramide, which is a type of sphingolipids catalyzed by neutral sphingomyelinase (nSMase) from sphingomyelin. More specifically, EV release can be blocked by the inhibition of the nSMase pathway $[4,5]$. GW4869, a nSMase inhibitor, significantly reduces the release of EVs and corresponding neurotoxicity in the HIV-infected, as well as immuneactivated macrophages and microglia, and in $\mathrm{AD}$ models in vitro and in vivo $[3,6,7]$.

Glutamine is the most abundant amino acid in the plasma, and the metabolism of glutamine involves hydrolysis to glutamate by mitochondrial enzyme glutaminase 1 (GLS1). Subsequently, glutamate can be excreted or can be further metabolized to $\alpha$-ketoglutarate. Our previous studies uncovered a pathogenic role of glutaminase (GLS) in neuroinflammation and neuronal injury. There are two major types of GLS identified in mammals, the "kidney-type" glutaminase (GLS1) and "liver-type" glutaminase. GLS1 is the predominant glutamine-utilizing enzyme in the central nervous system (CNS), where "livertype" glutaminase is expressed at a lower level [8]. GLS1 is known to be associated with cancer cell research and CNS diseases [9-11]. Due to tissue-specific alternative splicing from the same gene, two isoforms of GLS1 were identified in the human brain, kidney-type glutaminase (KGA) and glutaminase $\mathrm{C}(\mathrm{GAC})$. Both KGA and GAC appear to catalyze glutamine deamination with comparable enzyme efficacies and kinetics [12, 13]. GAC is upregulated in HIV-associated dementia brain samples and is also released in the conditioned medium from HIV-1-infected macrophages. Furthermore, mitochondrial stress during HIV infection leads to membrane destabilization and release of GLS from the mitochondrial matrix to the cytosol through the permeability transition pore $[14,15]$.

GLS1 has been identified as an important metabolic factor controlling EV release from astrocytes during neuroinflammation [16]. However, the mechanism by which GLS1 regulates EV biogenesis and release remains unknown. In this study, we determine the mechanism of EV biogenesis and release through GLS1-mediated glutamine metabolism in human macrophages. We further identify two key downstream metabolites- $\alpha$-ketoglutarate and ceramide-as critical factors regulating EV release during HIV-1 infection and immune activation. These studies may help understand how glutamine metabolism regulates $\mathrm{EV}$ release in the context of infection and inflammation.

\section{Results}

\section{GLS1 overexpression increases EV release in vitro}

To further study the functional impacts of GLS1 on EV release, we constructed adenovirus vectors that overexpressed KGA or GAC to mimic the upregulation of these isoforms during HIV-1 infection. The extracellular levels of glutamate increased significantly in both KGA- and GACoverexpressing HeLa cells at the multiplicities of infection (MOI) of 200 compared with those in uninfected or vectortreated cells (Additional file 1: Figure S1A). An MOI of 200 was then used for the following GLS1-overexpression experiments. The overexpression of KGA and GAC was confirmed by Western blot (Additional file 1: Figure S1B). GLS1 enzyme activity assay confirmed the increase in KGA and GAC activity in protein lysates from KGA- and GACoverexpressing HeLa cells, suggesting that the overexpressed KGA and GAC were functional (Additional file 1: Figure S1C). Consistent with GLS1 enzyme activity, the levels of intracellular glutamate (Additional file 1: Figure S1D) and extracellular glutamate (Additional file 1: Figure S1E) were increased after KGA and GAC overexpression compared with those in the GFP and control groups.

After confirming KGA and GAC overexpression, we investigated GLS1 activities in the extracellular fluid. We found that cell-free supernatants showed increased glutamate production after the addition of glutamine, indicating that GLS1 overexpression leads to elevated levels of GLS1 activities in the extracellular fluid. L-DON, a GLS1 inhibitor, blocked the excess generation of glutamate in the supernatants, confirming that the observed GLS1 activity in the extracellular supernatants was from the protein GLS1 (Fig. 1a). To confirm that the observed GLS1 activity in the cell-free supernatants was from EVs, we isolated EVs from cell-free supernatants and tested the EVs in GLS1 activity assay. EVs that were isolated from KGA- or GAC-overexpressing HeLa cells generated significantly higher levels of GLS1 when incubated with glutamine (Fig. 1b). When L-DON was added to the enzyme reactions, the generation of glutamate was blocked, suggesting that KGA and GAC overexpression induced EV release that contains high levels of GLS1 activities (Fig. 1b). To determine the EV regulation after KGA and GAC overexpression, proteins from EVs were subjected to Western blot for EV markers, including tTG and flotillin-2. The levels of tTG and flotillin-2 did not change in the whole cell lysates but specifically 

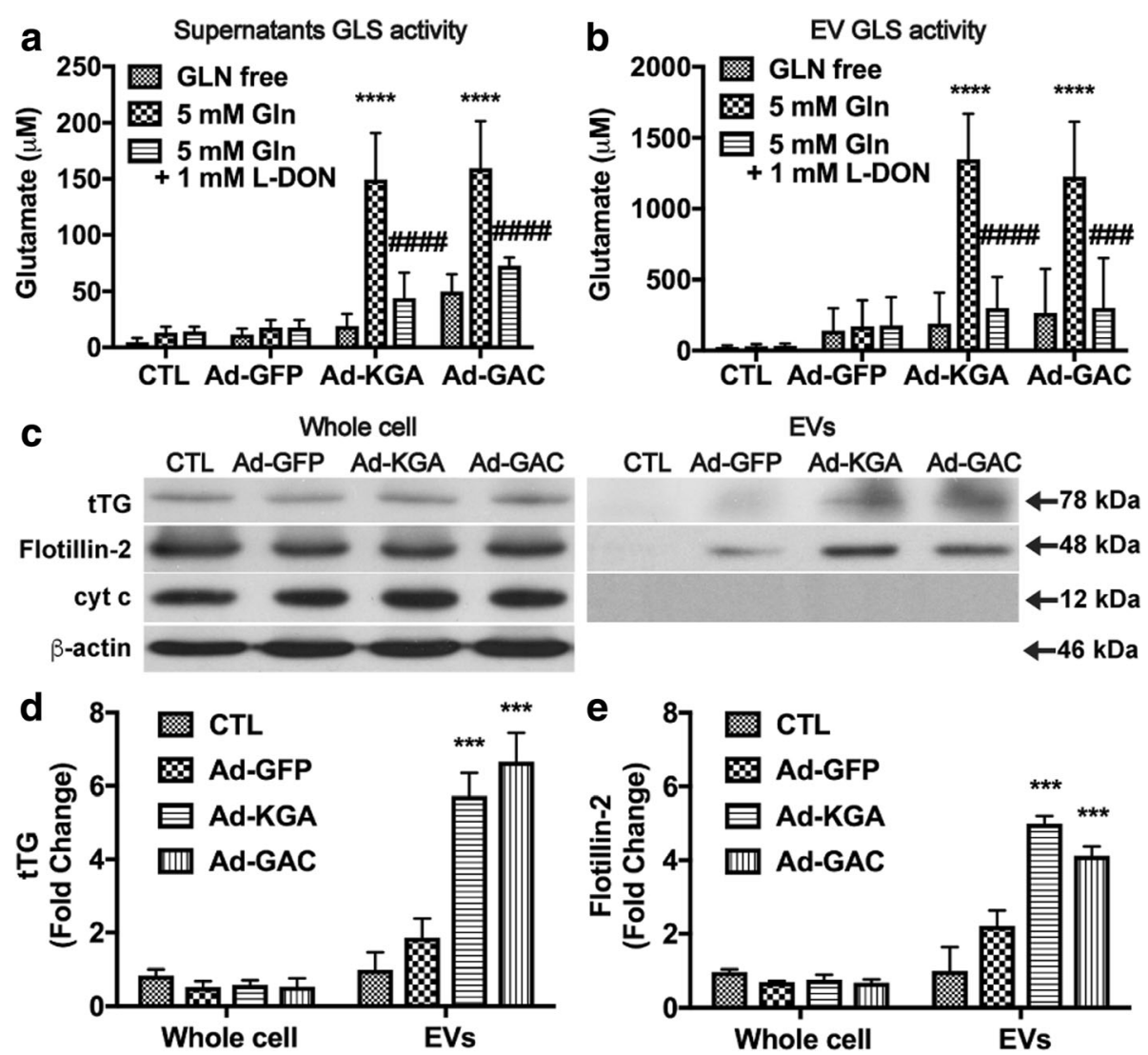

Fig. 1 KGA and GAC overexpression increase EV release in vitro. a Cell-free supernatants from control (CTL), Ad-GFP-, Ad-KGA-, and Ad-GAC-infected HeLa cells were incubated with or without $5 \mathrm{mM}$ glutamine (GIn) and $1 \mathrm{mM} \mathrm{L-DON}$ ex vivo for 2 days. The glutamate levels were determined by RP-HPLC. $\mathbf{b}$ EVs were isolated from cell-free supernatants from four different groups of HeLa cells and incubated with or without $5 \mathrm{mM}$ glutamine (Gln) and $1 \mathrm{mM}$ L-DON ex vivo for 2 days. Glutamate production from EVs was determined by RP-HPLC. c Protein lysates were prepared from the whole cell lysates and EV pellets. The levels of EVs markers, tTG and flotillin-2, were analyzed by Western blot. EV protein loading was normalized with protein concentrations in whole cell lysates. Mitochondrial marker, cytochrome c, was used as a control to exclude contamination of EV pellets. $\mathbf{d}$, e Densitometric quantifications of the protein levels in EVs were presented as fold change relative to that in control EV lysate. The protein levels in the whole cell lysates were presented as fold change relative to $\beta$-actin in the whole cells. Western blot results shown are representative of three independent experiments. CTL, control. Quantification results were normalized to the control group; ANOVA and post-test were performed on the remaining groups. ${ }^{* * *}$ and ${ }^{* * *}$ denote $p<0.001$ and 0.0001 , respectively, compared with the Ad-GFP group. ${ }^{\# \# \# ~ a n d ~}{ }^{\# \# \# ~}$ denote $p<0.001$ and 0.0001 , respectively, compared with the $5 \mathrm{mM}$ Gln group, $n=3$ per group

increased in EVs collected from KGA- and GACoverexpressed HeLa cells (Fig. 1c-f). The mitochondrial protein cytochrome $\mathrm{c}$ was absent in the EV preparation, suggesting that the preparation was not contaminated with subcellular organelles such as mitochondria. Together, these data suggest that elevation of GLS1 is sufficient to induce the extracellular release of EVs.

\section{EV release in HIV-1-infected macrophages is dependent} on glutamine metabolism

Our previous work demonstrated the role of GLS1 in the regulation of EV release [17]. However, the mechanism of how GLS1 regulates the release remains unclear. To determine whether glutamine metabolism is essential for EV release, we added different concentrations of glutamine to HIV-1-infected macrophages for 1 day and then isolated
EVs from the cell-free supernatants. The EVs were first subjected to negative staining TEM for characterization. EVs manifested typical morphologies in TEM and had sizes ranged from 40 to $300 \mathrm{~nm}$ (Additional file 1: Figure S2A-D). Quantification of EVs showed a significant increase of EVs at a concentration of $1 \mathrm{mM}$ and higher concentrations of glutamine did not appear to further increase EV release (Additional file 1: Figure S2E). Next, the addition of glutamine was confirmed with measurements of glutamate and glutamine in cell-free supernatants by reversed-phase highperformance liquid chromatography (RP-HPLC, Fig. 2a, b). To determine the EV regulation after glutamine treatment, we collected protein lysates from both the whole cells and EVs. The expression levels of GAC under different concentrations of glutamine did not change compared to those in control group (Fig. 2c). EV markers Alix and flotillin-2 did 

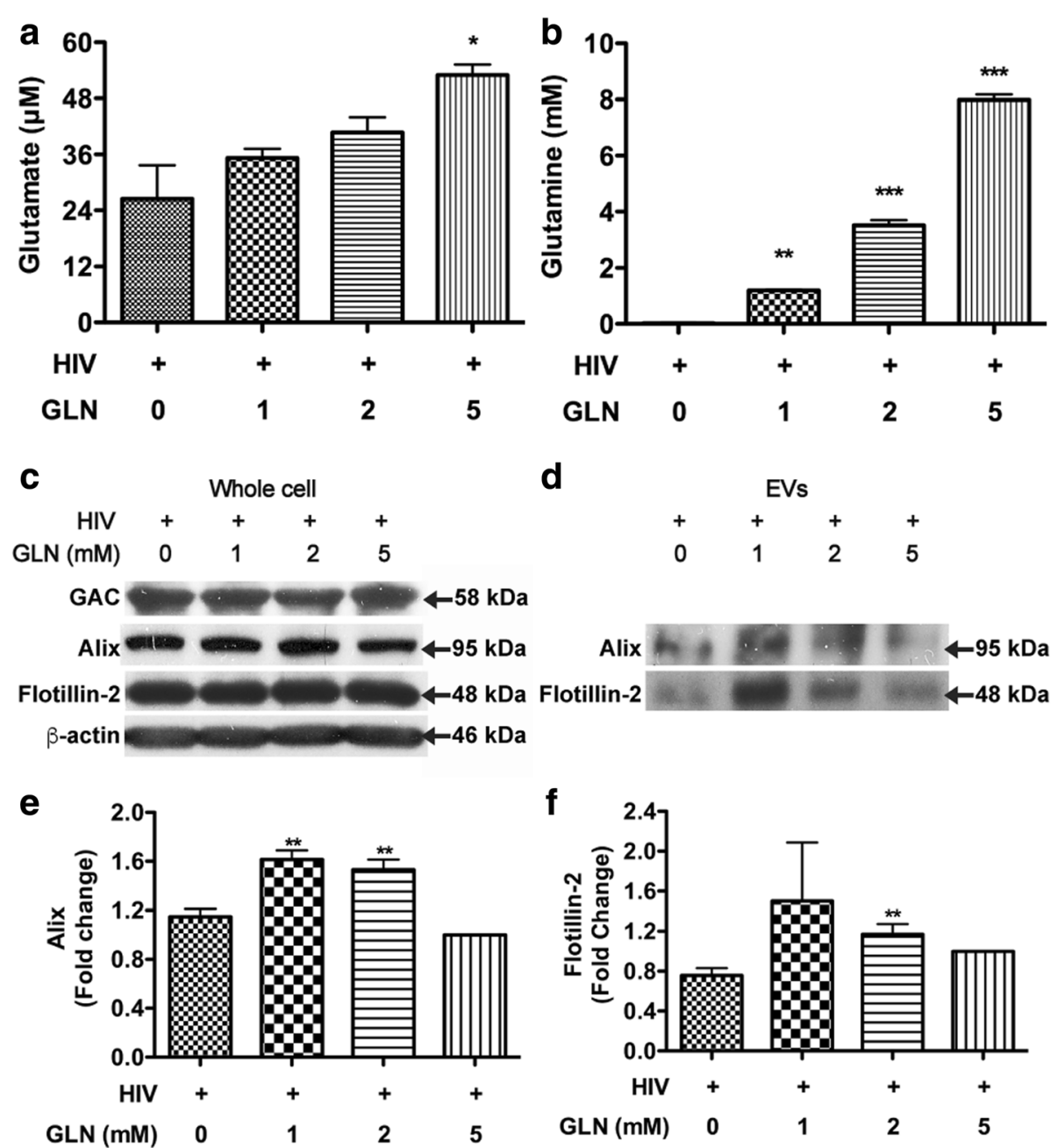

Fig. 2 EV release in HIV-1-infected macrophages is dependent on glutamine metabolism. MDM was infected by HIV-1 virus for 6 days, and the medium was changed into serum-free glutamine-free DMEM. Additional glutamine was added to the medium at the concentrations of 1, 2, and $5 \mathrm{mM}$. $\mathbf{a}, \mathbf{b}$ Supernatants were collected and centrifuged at $1500 \mathrm{rpm}$ for $5 \mathrm{~min}$ to remove cells. Samples were prepared for RP-HPLC, and the levels of glutamate and glutamine were determined. c, d Protein lysates were prepared from the whole cell lysates (c) and EVs pellets (d). The levels of EVs markers Alix and flotillin-2 in EVs, as well as the levels of $\beta$-actin in the whole cells, were determined by Western blot. EV protein loading was normalized with protein concentrations in the whole cell lysates. e, $\mathbf{f}$ Densitometric quantifications of the protein levels of EV markers were presented as fold changes relative to that in mock-infected control EV lysates. Western blot results shown are representative of the three independent experiments. Quantification results were normalized to the glutamine $5 \mathrm{mM}$ group; ANOVA and post-test were performed on the remaining groups. ${ }^{* * *}$, and ${ }^{* * *}$ denote $p<0.05,0.01$, and 0.001 , respectively, compared with that of the control microglia cells, $n=3$ per group.

not change in whole cell lysates but specifically increased in glutamine treatment groups at 1 and $2 \mathrm{mM}$ compared to controls (Fig. 2c-f). Glutamine treatment at $5 \mathrm{mM}$ did not induce significantly higher levels of EV release compared with the control. Together, these results suggest that EV release in HIV-1-infected macrophages is dependent on glutamine metabolism.

EV release in immune-activated microglia is dependent on glutamine metabolism

To investigate whether EV release is dependent on glutamine in microglia, a BV2 microglial cell line was used. Different concentrations of glutamine were added to the cultures $6 \mathrm{~h}$ prior to lipopolysaccharide (LPS) treatment. After overnight treatment, the levels of extracellular glutamate were increased with the addition of glutamine in a dose-dependent manner (Fig. 3a, b). To determine the EV regulation, protein lysates from whole cells and EVs were subjected to Western blots. In the whole cells, GAC showed a trend of increase with the addition of glutamine concentrations, whereas EV markers remained unchanged (Fig. 3c). In EV lysates, EV markers Alix and flotillin-2 were both significantly increased with the addition of glutamine (Fig. 3d-f). Consistent with Western blot analysis, nanoparticle tracking analysis (NTA) showed that 

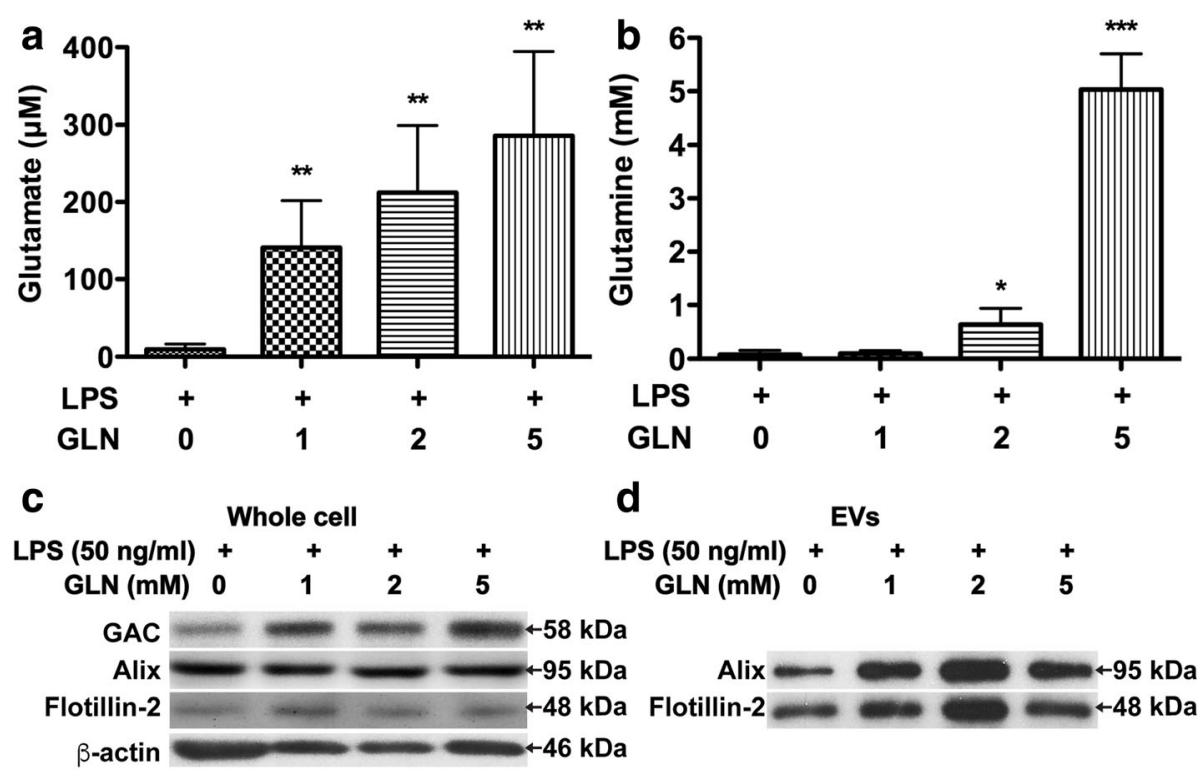

e
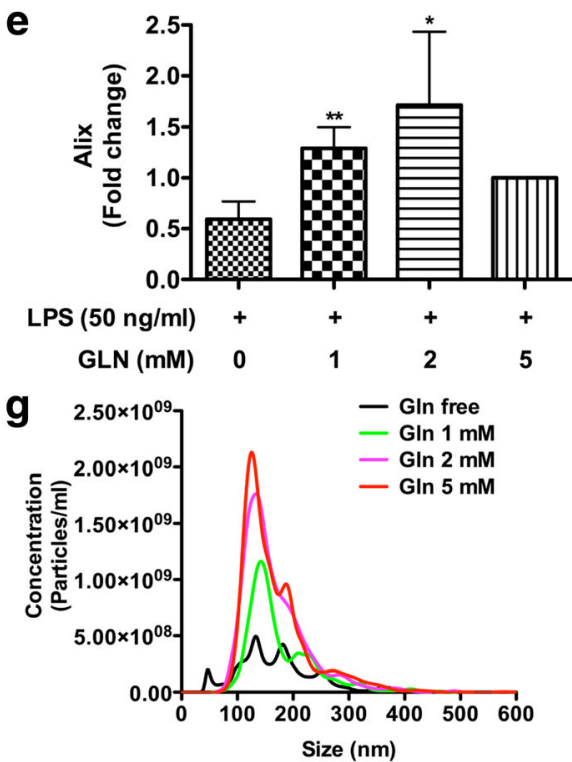

f

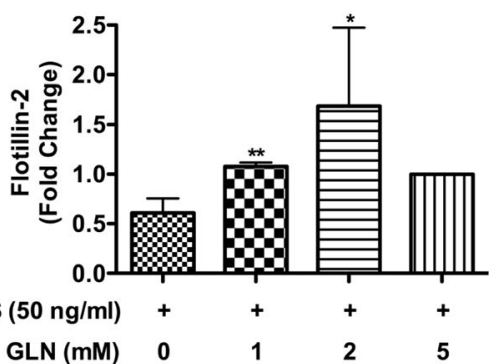

h

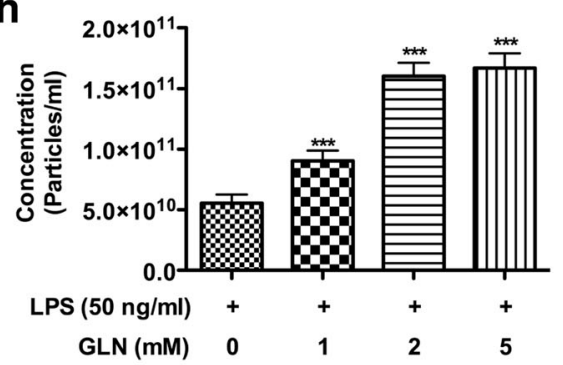

Fig. 3 EV release in immune-activated microglia is dependent on glutamine metabolism. When BV2 medium was changed into serum-free glutamine-free DMEM, additional glutamine was added to the medium at concentrations of 1, 2, and $5 \mathrm{mM} 6 \mathrm{~h}$ prior to LPS treatment. $\mathbf{a}$, $\mathbf{b}$ Supernatants were collected and centrifuged at $1500 \mathrm{rpm}$ for $5 \mathrm{~min}$ to remove cells. Samples were prepared for RP-HPLC, and the levels of glutamate and glutamine were determined. c, $\mathbf{d}$ Protein lysates were prepared from the whole cell lysates and EVs pellets. The levels of EVs markers Alix and flotillin-2 in EVs, as well as the levels of GAC and $\beta$-actin in whole cells, were determined by Western blot. EVs protein loading was normalized with protein concentrations in the whole cell lysates. $\mathbf{e}$, $\mathbf{f}$ Densitometric quantifications of the protein levels in EV markers were presented as fold changes relative to that in mock-infected control EV lysates. Western blot results shown are representative of the three independent experiments. Quantification results were normalized to the glutamine $5 \mathrm{mM}$ group; ANOVA and post-test were performed on the remaining groups. ${ }^{* * *},{ }^{* * *}$, and ${ }^{* * * *}$ denote $p<0.05,0.01,0.001$, and 0.0001 , respectively, compared with that of control Gln-free-treated microglia cells, $n=3$ per group (a-f). $\mathbf{g}$ EVs were isolated from culture supernatants and visualized through NanoSight. $\mathbf{h}$ Quantifications of NanoSight NTA of vesicle concentration for samples from LPS-treated BV2 cell with different glutamine concentrations. ANOVA and Bonferroni post-test; ${ }^{*}, * * * * *$, and ${ }^{* * *}$ denote $p<0.05,0.01,0.001$, and 0.0001 , respectively, compared with that of control Gln-free-treated microglia cells, $n=5$ per group

addition of glutamine significantly increased EV release in a dose-dependent manner (Fig. 3g, h). Altogether, these results demonstrated that EV release in immuneactivated microglia is dependent on glutamine.
Inhibition of GLS1 activity reduces EV release in HIV-1infected macrophages and immune-activated microglia To further investigate whether GLS1-mediated glutamine metabolism is crucial for EV release in HIV-1- 
infected macrophages and immune-activated microglia, we used BPTES, a potent GLS1 inhibitor [18]. Human macrophages were infected with HIV-1 virus with $10 \mu \mathrm{M}$ BPTES prior to sample collections (Fig. 4a). When BPTES was added 1 day prior to EV isolation, EV lysates showed significantly decreased levels of EV markers Alix, Flotillin-2, and tTG compared with those from HIV-1-infected macrophages (Fig. 4b-e). In contrast, when BPTES was added immediately after the HIV-1 infection, the EV regulation was not affected, indicating that BPTES affects the EV release within a limited time frame.

To investigate GLS1-mediated glutamine metabolism in immune-activated microglia, we used BPTES and CB839, both of which are potent GLS1 inhibitors [19]. BPTES or CB839 was added to BV2 cells prior to LPS treatment, and EV regulation was determined through Western blot and NTA (Fig. 5a). LPS, BPTES, and CB839 treatment did not significantly change BV2 cell viability (Additional file 1: Figure S3). In contrast, both levels of Alix and flotillin-2 were significantly increased after LPS stimulation in the EVs isolated from BV2 cells, suggesting that LPS increases EV release in BV2 cells (Fig. 5b-d). Pretreatment with BPTES and CB839 reduced the levels of Alix and flotillin-2 in LPS-activated BV2 cells (Fig. 5b-d). Consistent with Western blot data, NTA revealed that treatment with BPTES and CB839 significantly reduced the EV particles in both the LPSactivated and unactivated control BV2 cells. Collectively, these results indicated that the release of EVs in HIV-1infected macrophages and LPS-treated microglia is dependent upon GLS1-mediated glutamine metabolism.

\section{EV release in immune-activated microglia is dependent on the production of a-ketoglutarate}

GLS1-mediated glutamine metabolism produces glutamate and subsequently $\alpha$-ketoglutarate $(\alpha-K G)$. To determine whether $\alpha-K G$ is required for $\mathrm{EV}$ biogenesis and release, $1 \mathrm{mM}$ of $\alpha$-KG was added to LPS-stimulated BV2 cells with GLS1 inhibitors. Treatment with BPTES and CB839 significantly reduced GLS1 activity in the presence of $1 \mathrm{mM} \alpha-K G$ (Fig. 6a), confirming that they are indeed GLS1 inhibitors. In the presence of $\alpha-K G$, BPTES and CB839 failed to decrease EV markers Alix

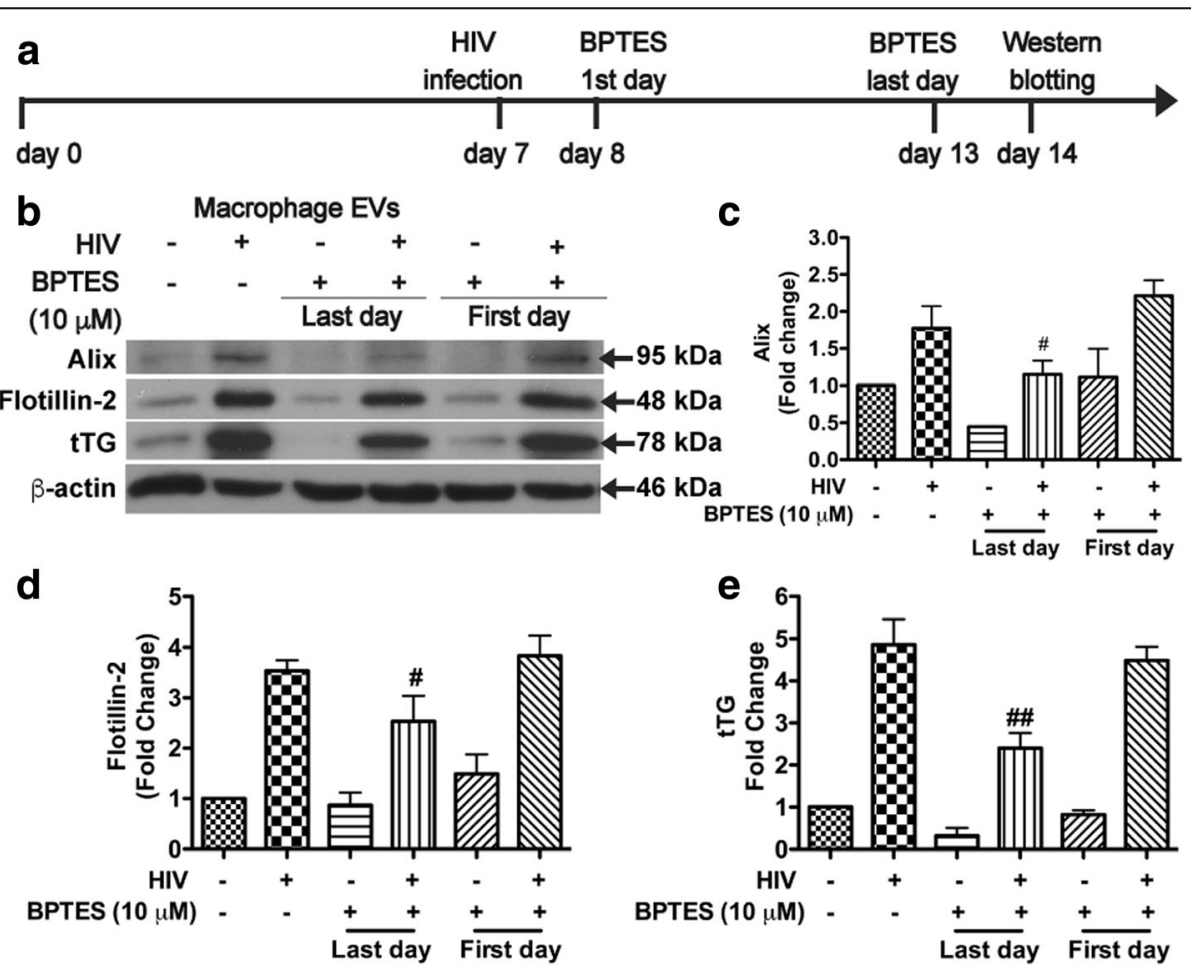

Fig. 4 Inhibition of glutaminase reduces EV release in HIV-1-infected macrophages. a Timeline of treatments of macrophages prior to collection. b EVs were isolated from mock-infected or HIV-1-infected macrophages treated with $10 \mu \mathrm{M}$ BPTES at the day after infection or 1 day prior to EV isolation. EV protein loading was normalized with protein concentrations in the whole cell lysates. EV markers, Alix, flotillin-2, and tTG in EVs, as well as the levels of $\beta$-actin in whole cells, were determined by Western blot. c-e Densitometric quantifications of the protein levels of Alix, flotillin-2, and tTG in EV markers were presented as fold change relative to that in mock-infected control EV lysates. Western blot results shown are representative of the three independent experiments. Quantification results were normalized to the control uninfected macrophage group; ANOVA and post-test were performed on the remaining groups. ${ }^{*}$ and ${ }^{* *}$ denote $p<0.05$ and $p<0.01$, respectively, compared with the HIV-1-infected macrophages, $n=3$ per group 

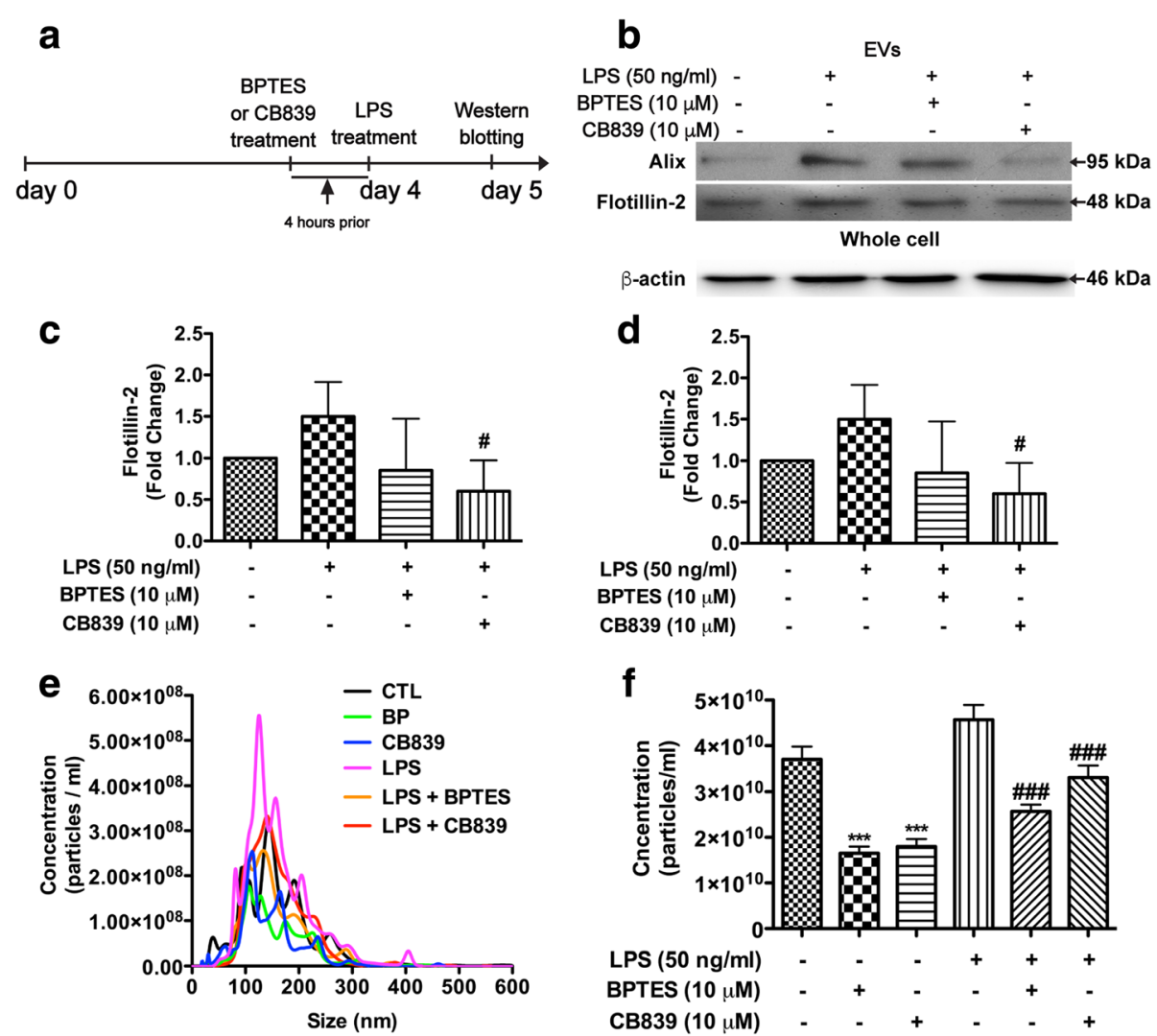

Fig. 5 Inhibition of glutaminase reduces EV release in immune-activated microglia. a BV2 cells were treated with $10 \mu M$ BPTES or $10 \mu M$ CB839 $4 \mathrm{~h}$ prior to LPS treatment overnight. BV2 cell regular medium was changed to serum-free DMEM medium when GLS1 inhibitors were added. b Protein lysates were prepared from EV pellets in control, LPS-treated, and LPS-treated with GLS1 inhibitor BV2 cells. The levels of EVs markers Alix and flotillin-2 in EVs, as well as the levels of $\beta$-actin in whole cells, were determined by Western blot. $\mathbf{c}$, $\mathbf{d}$ Densitometric quantification of the protein level of GAC in the whole cell lysates was presented as fold change relative to densitometric quantifications of the protein levels of in EV markers were presented as fold change relative to that in control EV lysates. Quantification results were normalized to the unactivated control BV2 group; ANOVA and post-test were performed on the remaining groups. ${ }^{\#}$ denotes $p<0.05$ compared with that of LPS-activated microglia group, $n=3$ per group. e EVs were isolated from culture supernatants and visualized through NanoSight. $\mathbf{f}$ Quantifications of the NanoSight NTA of vesicle concentration for samples from control and LPS-treated BV2 cells with or without BPTES and CB839. ANOVA and Bonferroni post-test; *** denotes $p<0.0001$ compared with that of the control BV2 cells. \#\#\# denotes $p<0.0001$ compared with that of the LPS-treated BV2 cells, $n=5$ per group

and flotillin-2 in EV lysates (Fig. 6b-d). Consistent with the Western blots, NTA revealed that suppression of EV release by BPTES and CB839 was reversed by $\alpha-K G$ (Fig. 6e, f). These results suggest that $\alpha-K G$ is the critical downstream effector that is responsible for the GLS1mediated EV release.

\section{GLS1 mediates EV release through sphingolipid metabolism}

Our previous report has shown that the release of EV can be blocked by GW4869, a nSMase inhibitor [6, 20]. nSMase is known to catalyze the production of ceramide, which is an active component of EVs. Therefore, we hypothesized that GLS1 mediates EV release through sphingolipid metabolism. GLS1 activity remained suppressed by BPTES and CB839 in the presence of $\mathrm{C}_{6}$ ceramide (Fig. 7a), a cell-permeable short-chain ceramide that can be supplied to cell cultures as exogenous ceramides to increase cell ceramide levels [21]. However, NTA showed that BPTES- and CB839-mediated inhibition of EV release was blocked by $\mathrm{C}_{6}$ ceramide (Fig. 7b), indicating that the ceramide pathway of sphingolipid metabolism is involved in the GLS1-regulated release of EVs.

\section{Brain-specific GLS1 overexpression increases EV release in vivo}

To determine the link between the GLS1 and the secretion of EVs in vivo, we used a GLS1 transgenic mouse model that we recently generated [17]. The transgenic mice have GLS1 isoform GAC overexpressed in all cells of the neural lineage. Positive mice were termed NestinGAC transgenic mice. We successfully isolated EVs from the Nestin-GAC transgenic brain tissues following the protocol from a previous publication [22]. After normalized EVs to equal amount of initial brain tissues used for EV isolation, we found that Nestin-GAC mice had 

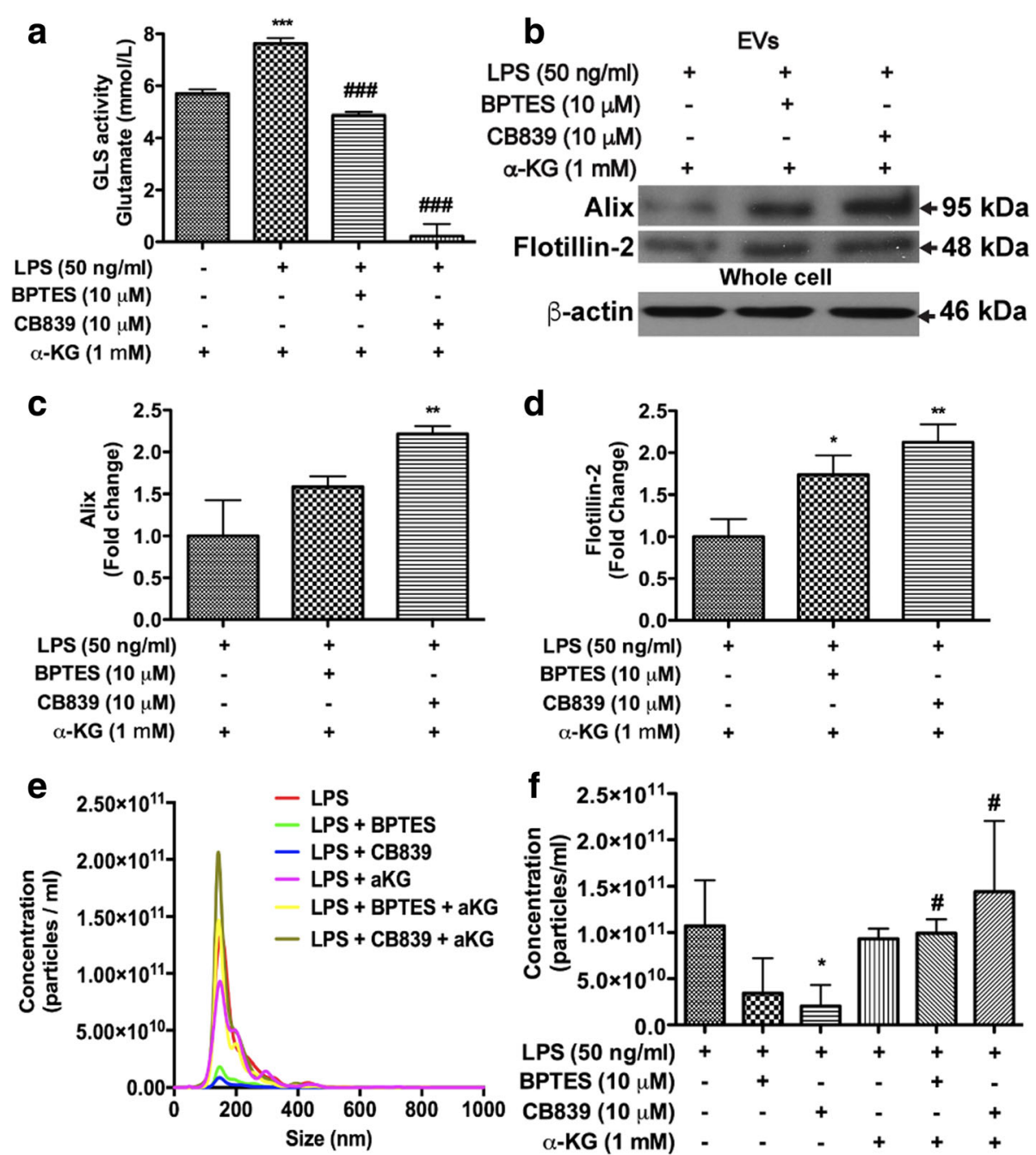

Fig. $6 \mathrm{EV}$ release in immune-activated microglia is associated with the level of a-ketoglutarate. BV2 cells were treated with $10 \mu \mathrm{M}$ BPTES or $10 \mu \mathrm{M}$ CB839 $4 \mathrm{~h}$ prior to LPS treatment overnight. BV2 cell regular medium was changed to serum-free DMEM medium when GLS1 inhibitors were added. Two hours prior to LPS treatment, $1 \mathrm{mM}$ of a-KG was added to BV2 cells. a Protein lysates were collected from LPS-treated BV2 cells with or without GLS1 inhibitors or a-KG. GLS1 activities were determined by the enzyme activity assay. b Protein lysates were prepared from EV pellets. The levels of EVs markers Alix and flotillin-2 in EVs, as well as the levels of $\beta$-actin in whole cells, were determined by Western blot. EV protein loading was normalized with protein concentrations in the whole cell lysates. $\mathbf{c}$, $\mathbf{d}$ Densitometric quantifications of the protein levels of in EV markers were presented as fold change relative to that in mock-infected control EV lysates. ANOVA and Bonferroni post-test; ${ }^{*}$ and ${ }^{* *}$ denote $p<$ 0.05 and 0.01 , respectively, compared with that of the control microglia cells, $n=3$ per group. e After $1 \mathrm{mM}$ of a-KG treatment, NTA was conducted with 100x dilution of collected EVs with filtered PBS. $\mathbf{f}$ Quantifications of the NanoSight NTA of vesicle concentration for samples from LPS-treated BV2 cell with GLS1 inhibitors and $\mathrm{a}-\mathrm{KG}$. ANOVA and Bonferroni post-test; ${ }^{*}$ denotes $p<0.05$ compared with that of the LPS-treated BV2 group. " denotes $p<0.05$ compared with the corresponding GLS1 inhibitor group without $a-K G, n=5$ per group

significantly higher levels of EV markers Alix and Flotillin-2 in the isolated EVs compared with those from control mice, suggesting that brain-specific overexpression of GLS increases EV release in vivo (Fig. 8a-c). To further characterize the EVs isolated from in vivo tissues, Thy1-GAC and wild-type (WT) were generated by crossing Thy1-Cre mice and CAG-loxp-GAC mice, and PCR reactions were performed to confirm the genotypes of the mice. EVs were isolated and purified from the hemibrains of both WT and Thy1-GAC mice and subjected to negative staining TEM analysis. The number of EVs was significantly higher compared with those in WT control (Fig. 8d-f). Together, these data indicate that the brain-specific GAC overexpression increases EV release in vivo.

\section{Discussion}

Glutamine is the most abundant amino acid in the plasma, and its metabolic products provide energy and substrates for a variety of biosynthesis pathways. During 

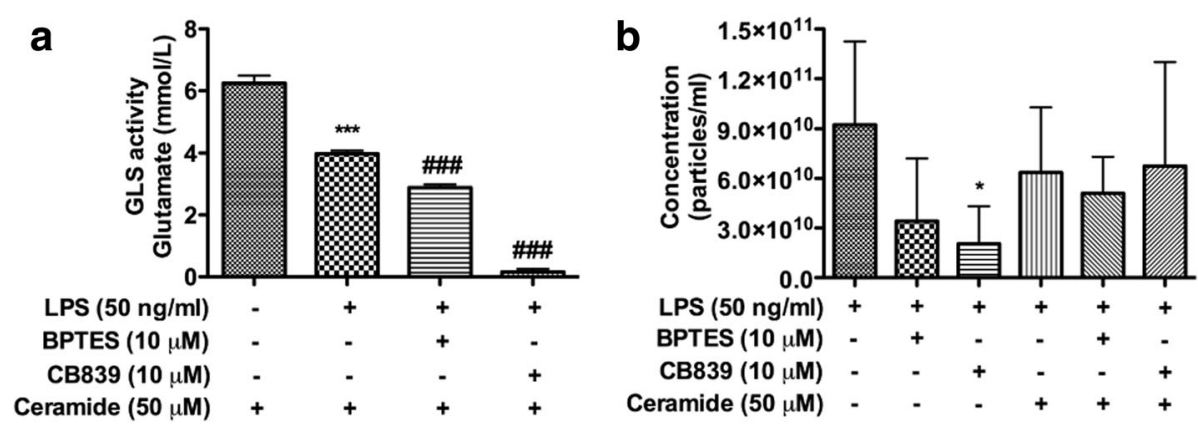

Fig. 7 EV release in immune-activated microglia is associated with sphingolipid metabolism. BV2 cells were treated with $10 \mu M$ BPTES or $10 \mu M$ CB839 $4 \mathrm{~h}$ prior to LPS treatment overnight. BV2 cell regular medium was changed to serum-free DMEM medium when GLS1 inhibitors were added. Two hours prior to LPS treatment, 50 or $100 \mu \mathrm{M}$ ceramide was added to BV2 cells. a Protein lysates were collected from LPS-treated BV2 cells with or without GLS1 inhibitors or ceramide. GLS1 activities were determined by the enzyme activity assay. ANOVA and Bonferroni post-test; *** denotes $p<0.001$ compared with that of the ceramide group. ${ }^{\# \#}$ denotes $p<0.001$ compared with that of the LPS-treated group, $n=3$ per group. b After 50- $\mu \mathrm{M}$ ceramide treatment, NTA was performed on the EVs isolated from the culture supernatants. Quantifications of the EV concentration were performed by NanoSight. ANOVA and Bonferroni post-test; * denotes $p<0.05$ compared with that of the LPS-treated group, $n=5$ per group

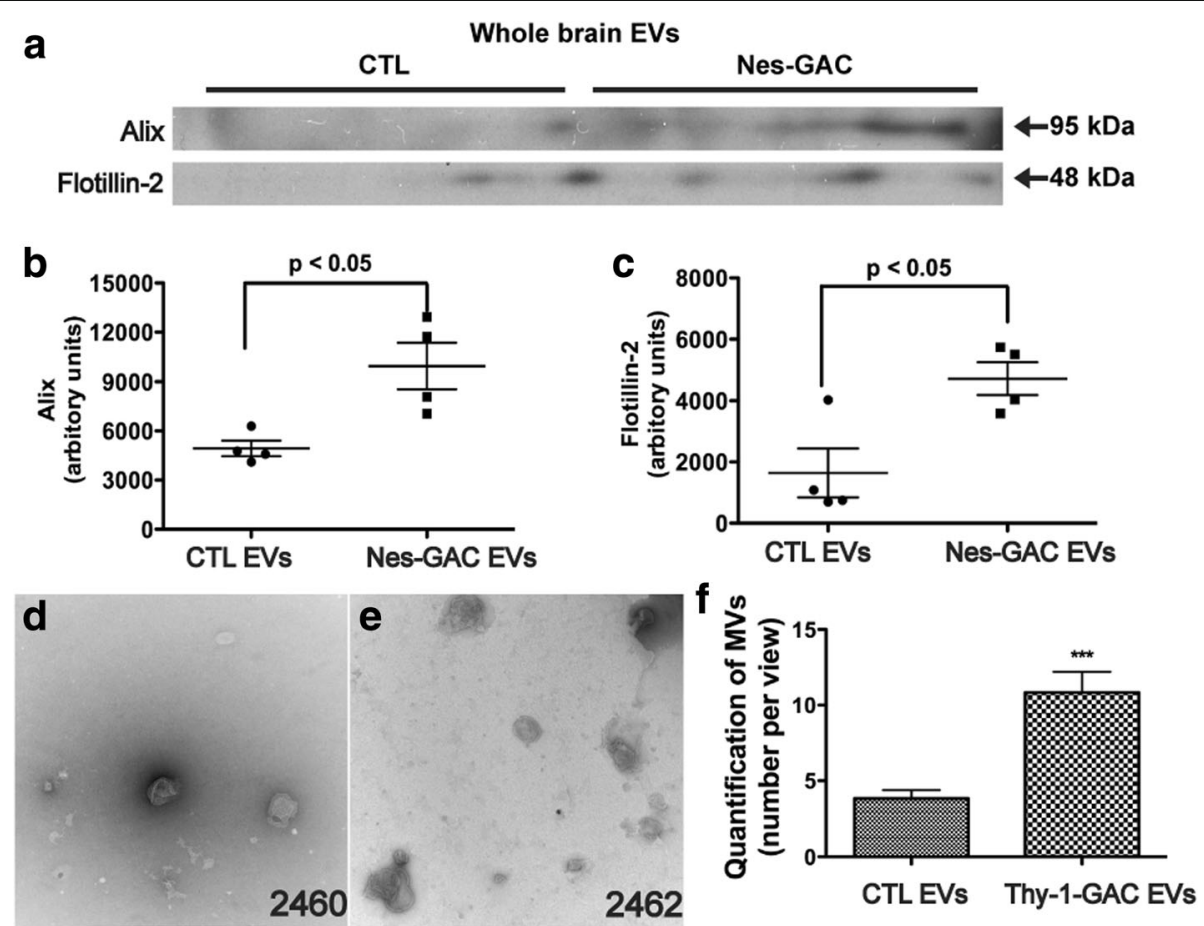

Fig. 8 Brain-specific GLS1 overexpression increases EV release in vivo. a Wild-type and Nestin-GAC mice were sacrificed at 12-16 weeks old. The brains were removed, and for each brain, the right hemibrain was processed for EVs isolation and the left hemibrain was homogenized for brain lysates. Protein lysates were prepared from the EVs pellets in wild-type and Nestin-GAC hemibrains. The levels of EVs markers, tTG and flotillin-2, in the EVs lysates were analyzed by Western blot. EV protein loading was normalized with the weight of their corresponding hemibrains before the EV isolation. $\mathbf{b}$, c Densitometric quantifications of the protein levels were presented as fold changes relative to that in wild-type EV lysate. Unpaired $t$ test, $n=4$ per genotype. Error bars are means \pm S.E.M. $\mathbf{d}-\mathbf{f}$ The brain tissues from adult Thy 1-GAC mice were dissected, and EVs were isolated using the same techniques described above. EVs collected from WT (d) and Thy 1-GAC (e) were evaluated via negative staining under TEM. Images shown were representative of each group. EV numbers per vision fields $(n=10)$ were quantified. CTL, control. Unpaired $t$ test, $* * *$ denotes $p<0.001$ 
viral infection and inflammation, glutamine metabolism is particularly important in that energy, biosynthesis, and antioxidative capacity are essential for a proper immune response. Our previous studies demonstrated a strong link between GLS and the neuropathogenesis of HIV-1 infection via the overproduction of neurotoxic levels of glutamate [11, 23-27]. Interestingly, GLS1 has been identified as an important metabolic factor controlling EV release from astrocytes in the presence of TNF$\alpha$ [16]. However, it remains unclear how glutamine metabolism regulates EV biogenesis and release. The current studies present a major finding regarding EV release. Upregulation of GLS1 induces an increase in the release of EVs through $\alpha-K G$ and ceramide in HIV-1infected macrophages and immune-activated microglia. The release of EVs is also observed to be increased in GAC-overexpressing transgenic mice. These new mechanistic regulations may help understand how glutamine metabolism shapes EV biogenesis and release during neuroinflammation.

Dysregulation of GLS1 has been reported in the pathogenesis of HIV-associated neurocognitive disorders and in cancer. In the CNS, GLS1 is a key enzyme in the glutamine metabolism, where glutamate, the main excitatory neurotransmitter, is produced to generate glutamate signaling and synaptic plasticity [28-32]. The dysregulation of GLS1 could potentially lead to the aberrant release of glutamate and compromise its neurotransmission. Indeed, abnormal glutamate neurotransmission is strongly associated with the memory loss and learning deficits due to the disrupted functioning of NMDA receptors [31-33]. To model upregulation of GLS1, we first constructed new adenoviruses overexpressing GLS1 isoforms. KGA- and GAC-overexpressing HeLa cells release a higher number of EVs into the supernatants. KGA- and GAC-mediated EV release can be blocked by GLS1 inhibitors BPTES and CB839, suggesting GLS1 is a critical factor regulating EV biogenesis and release. Similarly, we also used HIV-1infected macrophages and LPS-treated microglia to model GLS1 upregulation. HIV-1-infected macrophages and LPS-treated microglia have higher GLS1 levels compared to those of controls, which are associated with higher levels of EVs. Our third approach to model GLS1 upregulation is to use newly generated GAC transgenic mice. More EVs can be collected from GAC transgenic mice compared with those from negative littermates. However, it remains to be confirmed whether GLS1 inhibitors can block the EV release in vivo and whether the blocking of EV release could have protective effects on the GAC transgenic mice.

Our investigations reveal potential mechanisms of EV release in the context of HIV-1 infection and neuroinflammation. First, the release of EVs is dependent on the presence of glutamine. Second, $\alpha-K G$, a downstream product of glutamate, can rescue the inhibition of EV release by GLS1 inhibitors. Third, $\mathrm{C}_{6}$ ceramide, a cell-permeable analog of ceramide, can rescue the inhibition of $\mathrm{EV}$ release by GLS1 inhibitors. In glutamine repletion experiments, changes in EV release were observed at as low as $1 \mathrm{mM}$ of glutamine, the level of which is close to plasma concentration of glutamine [34]. However, the increase of glutamine concentration from 1 to $5 \mathrm{mM}$ did not show a dosedependent increase of EVs. Further testing of lower concentrations of glutamine will help to establish a dosedependent effect of glutamine. Another limitation of the current studies involves the experiments on $\alpha-K G$ and ceramide. It is unknown whether the effect of $\alpha-K G$ and $\mathrm{C}_{6}$ ceramide on EV release was direct or through other downstream metabolic intermediates. Given the variety of metabolic intermediates downstream of $\alpha-K G$, it is likely that other contributing factors in this pathway are required for EV biogenesis and release. Indeed, recent data suggest specific mechanisms controlled by glutaminolysis to fine-tune macrophage activities during both M2 and LPS activation involve a-KG and succinate $[35,36]$. EV biogenesis and release from macrophages may aid in the macrophage-mediated immune responses to infection. Therefore, harnessing EVs through glutamine/a-KG pathway would be an attractive strategy to regulate macrophage phenotypes in infection and inflammation.

Our characterizations of EVs involve the determination of EV markers in Western blots, EV size/concentrations in well-established NanoSight, and the morphological examination through negative staining TEM. Notably, the size of EVs remains the same throughout all the studies as determined by NanoSight analysis, indicating that the regulation of the EVs during HIV-1 infection and immune activation of macrophages/microglia are more specifically on the number and contents. EVs are known to include exosomes, microvesicles, and apoptotic bodies according to their cellular origin. In our studies, there is little evidence of apoptotic bodies in the EVs since the size of EVs was overwhelmingly smaller than $300 \mathrm{~nm}$, whereas typical apoptotic bodies are more than $500 \mathrm{~nm}$. Furthermore, it is known that EV isolation contains virions, and HIV-1 virions are around $145 \mathrm{~nm}$ [37], which may be mistaken as EVs during data interpretation. However, based on the data from NanoSight, EV quantities $\left(10^{8} \sim 10^{10} / \mathrm{ml}\right)$ greatly exceed the quantity of viral particles $\left(\sim 10^{6} / \mathrm{ml}\right)$ in the supernatant. Therefore, we conclude that the particles detected by the NanoSight are predominantly EVs, including exosomes and microvesicles.

Previously, we reported that using GW4869 could effectively inhibit the release of EVs in HIV-1-infected macrophages and LPS-treated microglia [6]. Studies have also shown that GW4869 could inhibit EV release in vitro and in vivo in $\mathrm{AD}$ models and prion diseases [3, 7, 38-42]. It has also been reported that sphingolipid metabolism could 
be involved in EV release in microglia and neurological diseases $[5,43]$. Our data demonstrate a causal role of ceramide in mediating GLS1-induced EV release. However, the exact role of sphingolipid metabolism in GLS1associated EV release remains to be fully elucidated. It is unclear whether other lipid pathways also involved in GLS-mediated EV regulation [44, 45]. Whether sphingolipid metabolism merely provides building blocks for EVs or more refined mechanisms are involved remains to be determined.

\section{Conclusions}

In summary, our studies suggest that GLS1-mediated glutamine metabolism is essential in regulating EV release during HIV-1 infection and immune activation. GLS1 regulates EV release through its key downstream products $\alpha$ ketoglutarate and ceramide. GLS1 overexpression in the brain leads to increased levels of EVs in vivo. These new mechanistic regulations may help understand how glutamine metabolism shapes EV release during infection and inflammation. Targeting glutamine/a-KG pathway would be an attractive strategy to regulate EV release.

\section{Methods}

\section{Ethics statement}

MDM were used in full compliance with the University of Nebraska Medical Center and National Institutes of Health ethical guidelines, with the Institutional Review Board (IRB) \#: 162-93-FB. We have the informed written consent from all participants involved in this study. All mice were housed and bred in the Comparative Medicine Animal Facilities at the University of Nebraska Medical Center. All procedures were conducted in accordance with the protocol (11-018-04) approved by the Institutional Animal Care and Use Committee at the University of Nebraska Medical Center.

\section{Culture, HIV-1 infection, and LPS activation of macrophages and microglia}

Human peripheral blood-derived mononuclear cells were isolated through leukopheresis from healthy donors. Human macrophages were differentiated in Dulbecco's modified Eagle's media (DMEM) (Sigma-Aldrich, St. Louis, MO) with $10 \%$ human serum, $50 \mu \mathrm{g} / \mathrm{ml}$ gentamycin, $10 \mu \mathrm{g} / \mathrm{ml}$ ciprofloxacin (Sigma), and $1000 \mathrm{U} / \mathrm{ml}$ recombinant human macrophage colony-stimulating factor (MCSF) for 7 days. Human fetal microglial cells were obtained from fetal brain tissue-derived microgliaastrocytes mixed cultures as previously described [46]. The HIV- $1_{\mathrm{ADA}}$ strain was used to infect the macrophages and microglia at a multiplicity of infection (MOI) of 0.1 and 0.5 , respectively. After $24 \mathrm{~h}$, the culture medium was changed to remove any remnant virus. Seven days after HIV-1-infection, the culture medium was changed to glutamine-free neurobasal medium for $24 \mathrm{~h}$, and supernatants were collected for subsequent HPLC or Western blot analysis. HeLa and $\mathrm{BV}_{2}$ cell lines were obtained from ATCC, and both cell lines were grown in DMEM with 10\% fetal bovine serum and antibiotics. LPS was used to immune activate $\mathrm{BV}_{2}$ cells for $24 \mathrm{~h}$, and supernatants were collected for HPLC and Western blot analysis. Bis-2-(5-phenylacetamido-1,2,4thiadiazol-2-yl)ethyl sulfide (BPTES) and CB839 (generous gifts provided by Dr. Takashi Tsukamoto from John Hopkins University and later ordered from Millipore with catalog numbers 530030 and 533717, respectively) were used in HIV-1-infected macrophages or LPStreated microglia prior to $\mathrm{EV}$ isolation. $\alpha$-Ketoglutarate (Sigma; 349631) and $C_{6}$ ceramide (Sigma; H6524) were also used to manipulate the metabolic intermediates in HIV-1-infected macrophages or LPS-treated microglia. All experiments involving human cell samples are approved by the Institutional Review Board at the University of Nebraska Medical Center.

\section{Adenoviral constructs}

Replication-defective adenovirus vectors expressing human KGA and GAC were generated using RAPAd ${ }^{\circ}$ CMV Adenoviral Expression System (Cell Biolabs, Inc., San Diego, CA). Generation of the full-length human KGA and GAC constructs was described in our prior publication [12]. Adenoviral constructs were amplified in a $293 \mathrm{AD}$ cell line (Cell Biolabs) and purified by ultracentrifugation through a $\mathrm{CsCl}$ gradient. Viral titer was determined by the Adeno- $\mathrm{X}^{\mathrm{mu}}$ Rapid Titer Kit (Clontech Laboratories, Inc., Mountain View, CA).

\section{Isolation of EVs from cells}

EVs were isolated from the supernatants of GLS1overexpressing cells, HIV-1-infected macrophages, and LPS-activated microglia through differential centrifugations. Briefly, the supernatants were first centrifuged at $300 \times g$ for $10 \mathrm{~min}$ to remove free cells, at $3000 \times g$ for $20 \mathrm{~min}$ to remove cellular debris, and then $10,000 \times g$ for $30 \mathrm{~min}$ to remove free organelles. Lastly, EVs were collected by ultracentrifugation at $100,000 \times g$ for $2 \mathrm{~h}$ at $4{ }^{\circ} \mathrm{C}$. To prepare EVs for Western blotting, the EV pellets were lysed in M-PER mammalian protein extraction reagent (Thermo Scientific, Pittsburgh, PA). For negative staining, EVs were fixed in $2 \%$ glutaraldehyde and $2 \%$ paraformaldehyde. For glutaminase activity assay and neurotoxicity, the EVs were resuspended in $1 \mathrm{ml}$ of glutamine-free neurobasal medium.

\section{Isolation of EVs from mice brain}

EV isolations from the brains were carried out as described previously with modifications according to the protocol [22]. The fresh and previously frozen mice hemibrains were harvested and dissected finely. The brain samples were then 
treated with 20 units/ml papain (Worthington) in Hibernate E solution (BrainBits, Springfield, IL) for $15 \mathrm{~min}$ at $37{ }^{\circ} \mathrm{C}$. The same volume of cold Hibernate $\mathrm{E}$ solution was added to the brain samples to stop the reaction of papain. The brain tissue was then gently homogenized and filtered through a $40-\mu \mathrm{m}$ mesh filter (BD Biosciences), followed by a centrifugation at $300 \times g$ for $10 \mathrm{~min}$ and $3000 \times g$ for $20 \mathrm{~min}$ at $4{ }^{\circ} \mathrm{C}$ to get rid of cells, membranes, and debris. After the supernatants were filtered through $0.45-\mu \mathrm{m}$ filter (Thermo Scientific), they were subjected to $10,000 \times g$ for $30 \mathrm{~min}$ at $4{ }^{\circ} \mathrm{C}$ to eliminate organelle contaminations. The supernatants were further centrifuged at $100,000 \times g$ for $70 \mathrm{~min}$ at $4{ }^{\circ} \mathrm{C}$ to pellet EVs. The pellets were then resuspended in filtered PBS, or MPER lysate solution for NanoSight or Western blot. All the samples were ultracentrifuged in ultraclear polycarbonate tubes (Beckman Coulter) that have a volume of $13.2 \mathrm{ml}$. A Beckman Coulter ultracentrifuge (Beckman Coulter OptimaL-90K ultracentrifuge; Beckman Coulter, Fullerton, CA, USA) was used with a rotor type SW $41 \mathrm{Ti}$.

\section{Negative staining and electron microscopy}

EVs were fixed and then spread on the silicon monoxide and nitro-cellular film-coated copper grid. The droplets were removed with filter paper, air-dried at room temperature, and then subjected to transmission electron microscopy (TEM).

\section{Nano-particle tracking analysis}

A NanoSight NS 300 (Malvern) equipped with an sCMOS camera was utilized to analyze the size distribution and concentration of EVs. NanoSight utilizes NTA, which is a combination of light scattering and Brownian motion technology to measure the concentration and size and distribution of particles in the EV supernatants. After the whole process of EV isolation, the pellets were first resuspended in $100 \mu \mathrm{l}$ of filtered PBS and then diluted 100 times. The conditions of the measurements include temperature of $25{ }^{\circ} \mathrm{C}$; viscosity of $1 \mathrm{cP}, 25 \mathrm{~s}$ per capture frame; and a measurement time of $60 \mathrm{~s}$. All the conditions were kept the same among all the samples. The results indicate the mean sizes and concentration of at least three individual measurements.

\section{Western blot}

Protein concentrations were determined by Bradford protein assay. SDS PAGE separated proteins from the whole cell and EV lysates. Afterward, they were electrophoretically transferred to polyvinyldifluoridene membranes (Millipore, Billerica, MA and Bio-Rad, Hercules, CA). The membranes were incubated overnight at $4{ }^{\circ} \mathrm{C}$ with polyclonal antibodies for KGA and GAC (Dr. N. Curthoys, Colorado State University, Fort Collins, $\mathrm{CO}$ ), tissue transglutaminase (tTG) (Lab Vision/Thermo, Fremont, CA), flotillin-2 (Cell Signaling Technology, Danvers, MA), and $\beta$-actin (Sigma), followed by horseradish peroxidase-linked secondary antirabbit or anti-mouse secondary antibodies (Cell Signaling Technology). Antigen-antibody complexes were visualized by Pierce ECL Western Blotting Substrate. For quantification of the data, films were scanned with a CanonScan 9950F scanner, and images were analyzed using the public domain NIH Image program (developed at the US National Institutes of Health).

\section{Glutaminase activity assay}

Highly concentrated whole cell lysates were collected from flasks and subjected to GLS activity assay using a two-step assay $[27,47]$. Briefly, protein concentrations in the lysates were tested by using BCA Protein Assay Kit (Pierce). All samples were normalized to same concentration. In the first step, $50 \mathrm{mg}$ of protein were added to $100 \mu \mathrm{l}$ of initial assay mix. The mix contains $50 \mathrm{mM}$ glutamine, $0.15 \mathrm{M}$ phosphate, $0.2 \mathrm{mM}$ EDTA, and $50 \mathrm{mM}$ Tris-acetate. The $\mathrm{PH}$ value of the mix was adjusted to 8.6 and incubated at $37{ }^{\circ} \mathrm{C}$ for $30 \mathrm{~min}$. Ten microliters of $3 \mathrm{~N}$ hydrochloric acid was added to inactivate the glutaminase activity and stop the reaction. In the second step, $1 \mathrm{ml}$ of the second reaction mix was added, which contained $0.4 \mathrm{mg}$ of purified bovine liver glutamate dehydrogenase (Sigma-Aldrich, St. Louis, MO, USA), $0.08 \mathrm{M}$ Tris-acetate at $\mathrm{pH}$ 9.4, $0.2 \mathrm{M}$ hydrazine, $0.25 \mathrm{mM}$ adenosine 5 '-diphosphate sodium salt, and $2 \mathrm{mM}$ $\beta$-nicotinamide adenine dinucleotide hydrate. The samples were mixed and incubated for $30 \mathrm{~min}$ at room temperature. One hundred microliters of the reaction was used for measurement, absorbance was determined at a wavelength of $340 \mathrm{~nm}$, and glutamate concentration was determined using a standard curve of 10, 5, 2.5, 1.25, 0.625, and $0.0 \mathrm{mM}$ glutamate, along with negative controls.

\section{Analysis of glutamate concentrations}

Glutamate levels were analyzed by RP-HPLC using an Agilent 1200 liquid chromatograph and fluorescence detector as previously described [14] with a few modifications. The experiments utilized $4.6 \times 75 \mathrm{~mm}, 3.5 \mu \mathrm{m}$ ZORBAX Eclipse AAA analytical columns (Agilent). A gradient elution program was optimized for glutamate measurement with a flow rate $0.75 \mathrm{ml} / \mathrm{min}$. The intracellular glutamate levels in the whole brain lysates of mice and whole cell lysates were determined by Amplex Red Glutamic Acid/Glutamate Oxidase Assay Kit (Invitrogen) based on the manufacturer's instruction. The brain tissue lysates and whole cell lysates were diluted to the same protein concentration before the assay.

\section{Statistical analysis}

Data are expressed as means \pm SD unless otherwise specified. Statistical analysis was performed using one-way analysis of variance (ANOVA), followed by the Bonferroni post-test for all paired observations unless otherwise 
specified. Significance was determined by a $p$ value $<0.05$. All experiments were performed with cells from at least three donors to account for any donor-specific differences. Assays were performed at least three times in triplicate or quadruplicate within each assay.

\section{Additional file}

Additional file 1: Figures S1. Both KGA and GAC are successfully overexpressed by adenovirus in vitro. S2: EV release in HIV-1-infected macrophages is dependent on glutamine. S3: LPS, BPTES, and CB839 do not affect BV2 cell viability. (DOCX $6796 \mathrm{~kb}$ )

\section{Abbreviations}

Alix: ALG-2 interacting protein; ANOVA: Analysis of variance; BPTES: Bis-2-(5phenylacetamido-1,3,4-thiadiazol-2-yl)ethyl sulfide; CNS: Central nervous system; EV: Extracellular vesicle; GAC: Glutaminase C; GLS1: Glutaminase 1; KGA: Kidney-type glutaminase; LPS: Lipopolysaccharide; MOI: Multiplicities of infection; nSMase: Neutral sphingomyelinase; NTA: Nanoparticle tracking analysis; RP-HPLC: Reversed-phase high-performance liquid chromatography; TEM: Transmission electron microscopy; tTG: Tissue transglutaminase; aKG: a-Ketoglutarate

\section{Acknowledgements}

We kindly thank Dr. Norman Curthoys for providing the KGA and GAC antibodies. We thank Dr. Changhai Tian, Dr. Santhi Gorantla, Ms. Li Wu, and Tom Bargar for the technical support of this work. Julie Ditter, Lenal Bottoms, Myhanh Che, Johna Belling, and Robin Taylor provided outstanding administrative and secretarial support.

\section{Funding}

This work was supported by grants from the National Key Basic Research Program of China (973Program Grant No. 2014CB965000, project 1 No. 2014CB965001 and project 3No. 2014CB965003), Innovative Research Groups of the National Natural Science Foundation of China (\#81221001 to JZ), and Joint Research Fund for Overseas Chinese, Hong Kong, and Macao Young Scientists of the National Natural Science Foundation of China (\#81329002 to JZ); National Institutes of Health: 2R56NS041858 (JZ), 1R01NS097195 (JZ), and R03 NS094071 (YH).

\section{Availability of data and materials}

Data sharing is not applicable to this article as no datasets were generated or analyzed during the current study.

\section{Significance statement}

Extracellular vesicles (EVs) are secretory vesicles that are abundant in the body and play an important role in cell-to-cell communication. In pathological conditions such as neuroinflammation, EVs are often upregulated. To understand how EVs are regulated during neuroinflammation, we use two cellular models to demonstrate that glutaminase 1-mediated glutamine metabolism, which is the main amino acid metabolic pathway, is essential for the biogenesis and release of extracellular vesicles. The molecular mechanism of such regulation appears to be on the production of a-ketoglutarate and its downstream intermediate ceramide. In line with these in vitro data, glutaminase 1 overexpression leads to increased levels of EVs in vivo. These new mechanistic regulations may help understand how glutamine metabolism shapes EV biogenesis and release during inflammatory diseases.

\section{Authors' contributions}

$B W, Y H$, and JZ designed the research. BW, JL, RZ, YL, JP, AB, LZ, YW, ZT, and $\mathrm{YH}$ performed theresearch and collected the data. BW, $\mathrm{YH}$, and JZ performed the data analysis and interpretation. $\mathrm{BW}, \mathrm{YH}, \mathrm{AB}$, and JZ wrote the paper. All authors read and approved the final manuscript.

\section{Consent for publication}

Not applicable.

\section{Competing interests}

The authors declare that they have no competing interests.

\section{Publisher's Note}

Springer Nature remains neutral with regard to jurisdictional claims in published maps and institutional affiliations.

\section{Author details}

${ }^{1}$ Center for Translational Neurodegeneration and Regenerative Therapy, Shanghai Tenth People's Hospital affiliated to Tongji University School of Medicine, Shanghai, China. ${ }^{2}$ Department of Pharmacology and Experimental Neuroscience, University of Nebraska Medical Center, Omaha, NE 68198, USA. ${ }^{3}$ Department of Pathology and Microbiology, University of Nebraska Medical Center, Omaha, NE 68198-5930, USA.

Received: 27 December 2017 Accepted: 7 March 2018

Published online: 14 March 2018

\section{References}

1. Joshi P, Turola E, Ruiz A, Bergami A, Libera DD, Benussi L, Giussani P, Magnani G, Comi G, Legname G, et al. Microglia convert aggregated amyloid-beta into neurotoxic forms through the shedding of microvesicles. Cell Death Differ. 2014:21:582-93.

2. Coleman BM, Hill AF. Extracellular vesicles - their role in the packaging and spread of misfolded proteins associated with neurodegenerative diseases. Semin Cell Dev Biol. 2015;40:89-96.

3. Asai H, Ikezu S, Tsunoda S, Medalla M, Luebke J, Haydar T, Wolozin B, Butovsky O, Kugler S, Ikezu T. Depletion of microglia and inhibition of exosome synthesis halt tau propagation. Nat Neurosci. 2015;18:1584-93.

4. Clarke CJ, Hannun YA. Neutral sphingomyelinases and nSMase2: bridging the gaps. Biochim Biophys Acta. 2006;1758:1893-901.

5. van Echten-Deckert G, Walter J. Sphingolipids: critical players in Alzheimer's disease. Prog Lipid Res. 2012;51:378-93.

6. Wu B, Huang Y, Braun AL, Tong Z, Zhao R, Li Y, Liu F, Zheng JC. Glutaminasecontaining microvesicles from HIV-1-infected macrophages and immuneactivated microglia induce neurotoxicity. Mol Neurodegener. 2015;10:61.

7. Guo BB, Bellingham SA, Hill AF. The neutral sphingomyelinase pathway regulates packaging of the prion protein into exosomes. J Biol Chem. 2015; 290:3455-67.

8. Baglietto-Vargas D, Lopez-Tellez JF, Moreno-Gonzalez I, Gutierrez A, Aledo JC. Segregation of two glutaminase isoforms in islets of Langerhans. Biochem J. 2004;381:483-7.

9. Erickson JW, Cerione RA. Glutaminase: a hot spot for regulation of cancer cell metabolism? Oncotarget. 2010;1:734-40.

10. Wang JB, Erickson JW, Fuji R, Ramachandran S, Gao P, Dinavahi R, Wilson KF, Ambrosio AL, Dias SM, Dang CV, Cerione RA. Targeting mitochondrial glutaminase activity inhibits oncogenic transformation. Cancer Cell. 2010;18:207-19.

11. Huang Y, Zhao L, Jia B, Wu L, Li Y, Curthoys N, Zheng JC. Glutaminase dysregulation in HIV-1-infected human microglia mediates neurotoxicity: relevant to HIV-1-associated neurocognitive disorders. J Neurosci. 2011; 31:15195-204

12. Li Y, Peer J, Zhao R, Xu Y, Wu B, Wang Y, Tian C, Huang Y, Zheng J. Serial deletion reveals structural basis and stability for the core enzyme activity of human glutaminase 1 isoforms: relevance to excitotoxic neurodegeneration. Transl Neurodegener. 2017;6:10.

13. Cassago A, Ferreira AP, Ferreira IM, Fornezari C, Gomes ER, Greene KS, Pereira HM, Garratt RC, Dias SM, Ambrosio AL. Mitochondrial localization and structure-based phosphate activation mechanism of Glutaminase C with implications for cancer metabolism. Proc Natl Acad Sci U S A. 2012;109:1092-7.

14. Tian C, Sun L, Jia B, Ma K, Curthoys N, Ding J, Zheng J. Mitochondrial glutaminase release contributes to glutamate-mediated neurotoxicity during human immunodeficiency virus-1 infection. J Neurolmmune Pharmacol. 2012;7:619-28.

15. Erdmann N, Tian C, Huang Y, Zhao J, Herek S, Curthoys N, Zheng J. In vitro glutaminase regulation and mechanisms of glutamate generation in HIV-1infected macrophage. J Neurochem. 2009;109:551-61.

16. Wang $K$, Ye L, Lu H, Chen H, Zhang Y, Huang Y, Zheng JC. TNF-alpha promotes extracellular vesicle release in mouse astrocytes through glutaminase. J Neuroinflammation. 2017;14:87. 
17. Wang Y, Li Y, Zhao R, Wu B, Lanoha B, Tong Z, Peer J, Liu J, Xiong H, Huang $Y$, Zheng J. Glutaminase $C$ overexpression in the brain induces learning deficits, synaptic dysfunctions, and neuroinflammation in mice. Brain Behav Immun. 2017:66:135-45.

18. Robinson MM, McBryant SJ, Tsukamoto T, Rojas C, Ferraris DV, Hamilton SK, Hansen JC, Curthoys NP. Novel mechanism of inhibition of rat kidney-type glutaminase by bis-2-(5-phenylacetamido-1,2,4-thiadiazol-2-yl)ethyl sulfide (BPTES). Biochem J. 2007:406:407-14.

19. Gross MI, Demo SD, Dennison JB, Chen L, Chernov-Rogan T, Goyal B, Janes JR, Laidig GJ, Lewis ER, Li J, et al. Antitumor activity of the glutaminase inhibitor CB-839 in triple-negative breast cancer. Mol Cancer Ther. 2014;13:890-901.

20. Huang Y, Li Y, Zhang H, Zhao R, Jing R, Xu Y, He M, Peer J, Kim Y, Luo J, et al. Zika virus propagation and release in human fetal astrocytes can be suppressed by neutral sphingomyelinase-2 inhibitor GW4869. Cell Discov. 2018; (In Press)

21. De Larichaudy J, Zufferli A, Serra F, Isidori AM, Naro F, Dessalle K, Desgeorges M, Piraud M, Cheillan D, Vidal H, et al. TNF-alpha- and tumor-induced skeletal muscle atrophy involves sphingolipid metabolism. Skelet Muscle. 2012;2:2.

22. Perez-Gonzalez R, Gauthier SA, Kumar A, Levy E. The exosome secretory pathway transports amyloid precursor protein carboxyl-terminal fragments from the cell into the brain extracellular space. J Biol Chem. 2012;287:43108-15.

23. Jiang Z, Piggee C, Heyes MP, Murphy C, Quearry B, Bauer M, Zheng J, Gendelman HE, Markey SP. Glutamate is a mediator of neurotoxicity in secretions of activated HIV-1-infected macrophages. J Neuroimmunol. 2001;117:97-107.

24. Zhao J, Lopez AL, Erichsen D, Herek S, Cotter RL, Curthoys NP, Zheng J. Mitochondrial glutaminase enhances extracellular glutamate production in HIV-1-infected macrophages: linkage to HIV-1 associated dementia. J Neurochem. 2004;88:169-80.

25. Huang $Y$, Erdmann N, Peng H, Zhao $Y$, Zheng J. The role of TNF related apoptosis-inducing ligand in neurodegenerative diseases. Cell Mol Immunol. 2005:2:113-22

26. Erdmann NB, Whitney NP, Zheng J. Potentiation of excitotoxicity in HIV-1 associated dementia and the significance of glutaminase. Clin Neurosci Res. 2006;6:315-28.

27. Erdmann N, Zhao J, Lopez AL, Herek S, Curthoys N, Hexum TD, Tsukamoto T, Ferraris D, Zheng J. Glutamate production by HIV-1 infected human macrophage is blocked by the inhibition of glutaminase. J Neurochem. 2007;102:539-49.

28. Yeckel MF, Berger TW. Spatial distribution of potentiated synapses in hippocampus: dependence on cellular mechanisms and network properties. J Neurosci. 1998;18:438-50.

29. Naie K, Manahan-Vaughan D. Regulation by metabotropic glutamate receptor 5 of LTP in the dentate gyrus of freely moving rats: relevance for learning and memory formation. Cereb Cortex. 2004;14:189-98.

30. Manahan-Vaughan D, Braunewell KH. The metabotropic glutamate receptor, mGluR5, is a key determinant of good and bad spatial learning performance and hippocampal synaptic plasticity. Cereb Cortex. 2005;15:1703-13.

31. Magnusson KR, Scruggs B, Zhao X, Hammersmark R. Age-related declines in a two-day reference memory task are associated with changes in NMDA receptor subunits in mice. BMC Neurosci. 2007;8:43.

32. Brim BL, Haskell R, Awedikian R, Ellinwood NM, Jin L, Kumar A, Foster TC, Magnusson KR. Memory in aged mice is rescued by enhanced expression of the GluN2B subunit of the NMDA receptor. Behav Brain Res. 2013;238:211-26.

33. Delint-Ramirez I, Salcedo-Tello P, Bermudez-Rattoni F. Spatial memory formation induces recruitment of NMDA receptor and PSD-95 to synaptic lipid rafts. J Neurochem. 2008;106:1658-68.

34. Helling G, Wahlin S, Smedberg M, Pettersson L, Tjader I, Norberg A, Rooyackers $\mathrm{O}$, Wernerman J. Plasma glutamine concentrations in liver failure. PLoS One. 2016;11:e0150440.

35. Jha AK, Huang SC, Sergushichev A, Lampropoulou V, Ivanova Y, Loginicheva E, Chmielewski K, Stewart KM, Ashall J, Everts B, et al. Network integration of parallel metabolic and transcriptional data reveals metabolic modules that regulate macrophage polarization. Immunity. 2015;42:419-30.

36. Liu PS, Wang H, Li X, Chao T, Teav T, Christen S, Di Conza G, Cheng WC, Chou CH, Vavakova M, et al. Alpha-ketoglutarate orchestrates macrophage activation through metabolic and epigenetic reprogramming. Nat Immunol. 2017;18:985-94.

37. Briggs JA, Wilk T, Welker R, Krausslich HG, Fuller SD. Structural organization of authentic, mature HIV-1 virions and cores. EMBO J. 2003:22:1707-15.

38. Dinkins MB, Dasgupta S, Wang G, Zhu G, Bieberich E. Exosome reduction in vivo is associated with lower amyloid plaque load in the 5XFAD mouse model of Alzheimer's disease. Neurobiol Aging. 2014;35:1792-800.
39. Fedele E, Rivera D, Marengo B, Pronzato MA, Ricciarelli R. Amyloid beta: walking on the dark side of the moon. Mech Ageing Dev. 2015;152:1-4.

40. Joshi P, Benussi L, Furlan R, Ghidoni R, Verderio C. Extracellular vesicles in Alzheimer's disease: friends or foes? Focus on abeta-vesicle interaction. Int J Mol Sci. 2015;16:4800-13.

41. Yuyama K, Sun H, Mitsutake S, Igarashi Y. Sphingolipid-modulated exosome secretion promotes clearance of amyloid-beta by microglia. J Biol Chem. 2012;287:10977-89.

42. Wang G, Dinkins M, He Q, Zhu G, Poirier C, Campbell A, Mayer-Proschel M, Bieberich E. Astrocytes secrete exosomes enriched with proapoptotic ceramide and prostate apoptosis response 4 (PAR-4): potential mechanism of apoptosis induction in Alzheimer disease (AD). J Biol Chem. 2012;287:21384-95.

43. Antonucci F, Turola E, Riganti L, Caleo M, Gabrielli M, Perrotta C, Novellino L, Clementi $E$, Giussani P, Viani $P$, et al. Microvesicles released from microglia stimulate synaptic activity via enhanced sphingolipid metabolism. EMBO J. 2012;31:1231-40.

44. Tamboli IY, Barth E, Christian L, Siepmann M, Kumar S, Singh S, Tolksdorf K, Heneka MT, Lutjohann D, Wunderlich P, Walter J. Statins promote the degradation of extracellular amyloid \{beta\}-peptide by microglia via stimulation of exosome-associated insulin-degrading enzyme (IDE) secretion. J Biol Chem. 2010;285:37405-14.

45. Parlo RA, Coleman PS. Enhanced rate of citrate export from cholesterol-rich hepatoma mitochondria. The truncated Krebs cycle and other metabolic ramifications of mitochondrial membrane cholesterol. J Biol Chem. 1984; 259:9997-10003

46. Chao CC, Gekker G, Sheng WS, Hu S, Tsang M, Peterson PK. Priming effect of morphine on the production of tumor necrosis factor-alpha by microglia: implications in respiratory burst activity and human immunodeficiency virus-1 expression. J Pharmacol Exp Ther. 1994;269:198-203.

47. Curthoys NP, Weiss RF. Regulation of renal ammoniagenesis. Subcellular localization of rat kidney glutaminase isoenzymes. J Biol Chem. 1974;249:3261-6.

\section{Submit your next manuscript to BioMed Central and we will help you at every step:}

- We accept pre-submission inquiries

- Our selector tool helps you to find the most relevant journal

- We provide round the clock customer support

- Convenient online submission

- Thorough peer review

- Inclusion in PubMed and all major indexing services

- Maximum visibility for your research

Submit your manuscript at www.biomedcentral.com/submit
) Biomed Central 